\title{
Influence of Molecular Weight on Critical Concentration of Chitosan/Formic Acid Liquid Crystalline Solution
}

\author{
Yanming Dong, ${ }^{\dagger}$ Weibi QIU, Yonghong RuAN, Yusong WU, Mian WANG, and Chongyi XU \\ Department of Materials Science and Engineering, State Key Laboratory for Physical Chemistry of Solid \\ Surfaces, Xiamen University, Xiamen 361005, China
}

(Received September 11, 2000; Accepted January 29, 2001)

\begin{abstract}
Chitosan samples with viscosity average molar mass $\bar{M}_{\mathrm{V}}$ from $1 \times 10^{4}$ to $58 \times 10^{4}$ were prepared by depolymerization using nitrous acid. In higher molar mass region, critical concentrations to form liquid crystal phase in formic acid corresponded with the predicted values of Flory theory. Significant increase of the critical concentration was observed with decrease of molar mass, especially in the lower molar mass region. The results are qualitatively consistent with the calculated values of Khokhlov-Semenov theory, but deviate from calculated values higher at low molar mass region. This was explained by the inaccuracy of the second Virial approximation basic to this theory at higher concentrations for chains with small persistence length $q$. A linear relationship between the critical volume fraction $V_{2}^{\prime}$ and $\log \bar{M}_{\mathrm{V}}$ was found for this system.
\end{abstract}

KEY WORDS Chitosan / Liquid Crystal / Molar Weight / Critical Concentration /

Chitosan is the deacetylated product of chitin, an abundant natural linear polymer of $\beta-(1 \rightarrow 4)$ poly- $N$ acetylglucosamine. Chitosan and its derivatives often display lyotropic liquid crystalline behavior. ${ }^{1-6}$ There are two major structure parameters of chitosanwhich affect the concentration required to form the ordered phase (critical concentration $C^{*}$ ), i. e., molecular weight and degree of deacetylation (D. D.).

There have been few studies on the influence of molar mass upon $C^{*}$ of chitosan. Terbojevich et al. reported that $C^{*}$ of chitosan in aqueous $10 \%$ acetic acid increases with the decrease of molar mass for four samples. ${ }^{3}$ Wang et al. studied three chitosan samples with different molar mass in dichloroacetic acid, and observed evident variation of $C^{*} .^{7}$ Recently $\mathrm{Hu}$ et al. reported slight increase of $C^{*}$ with decrease of molar mass of chitosan for six samples in dichloroacetic acid solutions. ${ }^{8}$ These works all investigate the region of high or medium molar mass $\left(10^{5}-10^{6}\right)$, but neglect the low molar mass region $\left(\sim 10^{4}\right)$ which is just the most sensitive region. So this paper concentrates on the variation rule of $C^{*}$ with molar mass from $1 \times 10^{4}$ to $58 \times 10^{4}$, An attempt is made to relate $C^{*}$ to stiffness of chitosan.

\section{EXPERIMENTAL}

Chitosan was purchased from Xiamen Second Pharmaceutical Factory, China. Chitosan samples with different molar mass were prepared by depolymerizing chitosan with nitrous acid according to the method of literatures. ${ }^{9,10}$ Molar mass was controlled by the concentration of $\mathrm{NaNO}_{2}$ and depolymerization time. Preparation conditions are listed in Table I.

Intrinsic viscosity numbers $[\eta]$ of these samples were determined in $0.1 \mathrm{~mol} \mathrm{~L} \mathrm{~L}^{-1} \mathrm{CH}_{3} \mathrm{COONa}+0.2 \mathrm{~mol} \mathrm{~L}-1$ $\mathrm{CH}_{3} \mathrm{COOH}$ buffer solution at $30^{\circ} \mathrm{C}$. Viscosity-average molar mass $\left(\bar{M}_{\mathrm{V}}\right)$ was calculated from intrinsic viscosity number using the parameters of Mark-Howink equation

${ }^{\dagger}$ To whom correspondence should be addressed.
$K=1.424 \times 10^{-3} \mathrm{~mL} \mathrm{~g}^{-1}$ and $\alpha=0.96$ for D. D. $=84 \% .^{11}$

D. D. was determined with a Nicolet Avatar FT-IR 360 spectroscopy using absorbance ratio $A_{1550} / A_{2878}{ }^{12}$

$C^{*}(\mathrm{wt} \%)$ in formic acid solution was determined using the method described previously ${ }^{6}$ with an Olympus polarized microscope at $20^{\circ} \mathrm{C}$. The critical volume fraction $V_{2}^{\prime}$ was calculated from $C^{*}$ with the density of chitosan $\left(1.5 \mathrm{~g} \mathrm{~mL}^{-3}\right)$ and formic acid $\left(1.22 \mathrm{~g} \mathrm{~mL}^{-3}\right) . C^{*}$ or $V_{2}^{\prime}$ represent the boundary concentration between isotropic and biphasic phases.

\section{RESULTS AND DISCUSSION}

Table II shows that D. D. of all samples are almost the same, so the samples can be used to compare the effects of molar mass. $V_{2}^{\prime}$ increased dramatically from $4.1 \%$ to $40.0 \%$ with decrease of molar mass. The results are consistent with data obtained by Terbojevich et al. ${ }^{3}$ (see Figure 1), although their D. D. and solvent are different.

Liquid crystal phase of rod-like chain is predicted to occur at $V_{2}^{\prime}$ given by Flory's well-known equation, ${ }^{13}$

$$
V_{2}^{\prime} \cong(8 / x)(1-2 / x)
$$

where $x$ is the axis ratio of the rods. $x$ have been deter-

Table I. Preparation conditions of chitosan with different molar masses

\begin{tabular}{ccc}
\hline Sample symbol & $\begin{array}{c}\text { Concentration of } \mathrm{NaNO}_{2} \\
\text { solution/g L }\end{array}$ & $\begin{array}{c}\text { Depolymerization } \\
\text { time/h }\end{array}$ \\
\hline $1^{\mathrm{a}}$ & - & - \\
2 & 18.3 & 0.025 \\
3 & 6.3 & 0.083 \\
4 & 7.8 & 0.167 \\
5 & 10.0 & 0.250 \\
6 & 13.1 & 0.333 \\
7 & 8.6 & 7.0 \\
8 & 7.3 & 14.5 \\
9 & 5.3 & 18.0 \\
\hline
\end{tabular}

${ }^{\mathrm{a}}$ Blank test (without $\mathrm{NaNO}_{2}$ ). 
Y. DoNG et al.

Table II. Characteristics of chitosan samples

\begin{tabular}{|c|c|c|c|c|c|c|}
\hline Sample symbol & D.D. $1 \%$ & $\begin{array}{l}\text { The content of } \\
\text { amino groups } / \%\end{array}$ & {$[\eta] \times 10^{-2} / \mathrm{mL} \mathrm{g}^{-1}$} & $\bar{M}_{\mathrm{V}} \times 10^{-4}$ & $C^{*} / \mathrm{w} / \mathrm{w} \%$ & $V_{2}^{\prime} / \mathrm{v} / \mathrm{v} \%$ \\
\hline 1 & 83.5 & 7.96 & 4.86 & 58.0 & 5 & 4.1 \\
\hline 2 & 82.4 & 7.83 & 3.77 & 44.6 & 9 & 7.4 \\
\hline 3 & 84.4 & 8.06 & 2.98 & 34.9 & 13 & 10.8 \\
\hline 4 & 82.8 & 7.88 & 2.25 & 26.0 & 13 & 10.8 \\
\hline 5 & 82.4 & 7.83 & 0.97 & 10.8 & 27 & 23.1 \\
\hline 6 & 82.8 & 7.88 & 0.71 & 7.86 & 29 & 24.8 \\
\hline 7 & 85.9 & 8.23 & 0.31 & 3.25 & 32 & 27.7 \\
\hline 8 & 82.8 & 7.88 & 0.15 & 1.57 & 40 & 35.2 \\
\hline 9 & 85.6 & 8.20 & 0.13 & 1.35 & 44 & 40.0 \\
\hline
\end{tabular}

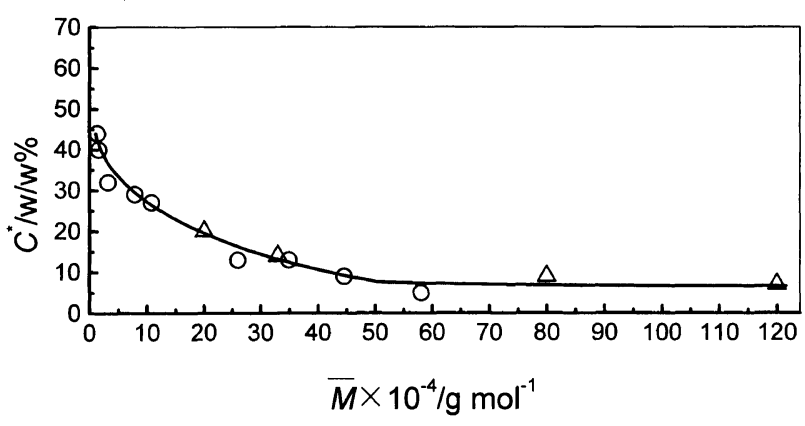

Figure 1. $C^{*}$ as function of molar mass. $(\triangle)$ are reference data ${ }^{3}$.

mined from either the contour length $L$ ( $2 L / d$ for rods) or persistence length $q$ ( $2 q / d$ for semirigid chains, where $d$ is the diameter of chain). If $q$ of chitosan $(q \approx 22 \mathrm{~nm})^{3}$ is adopted, the theoretical curve is drawn in Figure 2 to compare with experimental results. It can be observed that the results correspond with theoretical curve at higher molar mass region.

Khokhlov and Semenov ${ }^{14,15}$ used finite stiffness in their theoretical formulation of semi-flexible polymer, where they have extended the theory of Onsager ${ }^{16}$ for rods to worm-like chains. The persistence length $q$ of the chain plays a central role.

The expression deduced by Khokhlov and Semenov is as follows:

$$
\begin{gathered}
V_{2}^{\prime}=\frac{d}{2 q}\left[\frac{3.34+11.94(L / 2 q)+6.34(L / 2 q)^{2}}{[1+0.586(L / 2 q)](L / 2 q)}\right] \\
d=\left(M_{0} / \rho \times N_{\mathrm{A}} \times L_{0}\right)^{\frac{1}{2}}
\end{gathered}
$$

where $\rho$ is density of polymer, $M_{0}$ is molar mass of repeat unit, $N_{\mathrm{A}}$ is Avogadro constant and $L_{0}$ is the shadow length of repeat unit along chain (taken $0.515 \mathrm{~nm}$, half of $b$ in unit cell of chitosan crystal $^{2}$ ). $q=22 \mathrm{~nm}$ again. ${ }^{3}$

Calculated plots are shown in Figure 2. The experimental curve for chitosan is qualitatively consistent with the theoretical values. But $V_{2}^{\prime}$ had more molar mass dependence than theory anticipation, especially in lower molar mass region. In higher molar mass region, the experimental values were more close to calculated values of Flory theory than those of Khokhlov-Semenov theory.

Odijk ${ }^{17}$ has shown that the Khokhlov-Semenov theory agrees well with data on such slender polymer chains as schizophyllan, poly $(\gamma$-benzyl L-glutamate) (PBLG), and

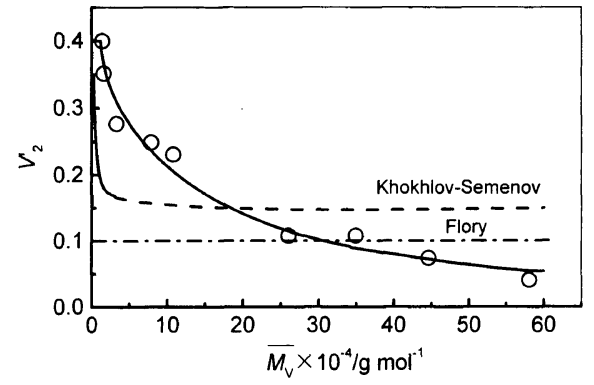

Figure 2. Relation between $V_{2}^{\prime}$ and $\bar{M}_{\mathrm{V}}$, compared with Flory theory curve (----) and Khokhlov-Semenov theory curve (-----) .

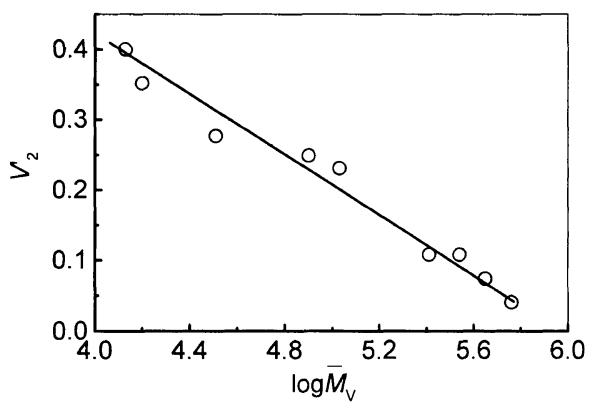

Figure 3. Plots of $V_{2}^{\prime}$ vs. $\log \bar{M}_{\mathrm{V}}$ for chitosan/formic acid system.

DNA. However, his analysis is limited to relatively rigid polymer, namely, those with larger $q$. Itou et al. ${ }^{18}$ found that this theory failed to explain the data for poly(hexyl isocyanate)/dichloromethane solutions, where $q$ was as small as $21 \mathrm{~nm}$. This suggests that the second Virial approximation basic to this theory would be inaccurate at higher concentrations. For the same reason, differentials between experimental data and calculated values for chitosan also can be attributed to small $q$.

Although no suitable theory can be used for relative flexible chain at low molar mass region, a linear relationship between $V_{2}^{\prime}$ and $\log \bar{M}_{\mathrm{V}}$ was found for chitosan/ formic acid solutions (Figure 3 ).

The equation of the curve is

$$
V_{2}^{\prime}=-0.2022 \log \bar{M}_{\mathrm{V}}+1.2184
$$

The dependence of molar mass on $C^{*}$ for chitosan/formic acid system seems to follow the logarithmic rule of molar mass.

Chitosan contains amino groups are ionizable in acid. Thus it is expected that chitosan exists as a polyelectro- 
Table III. $C^{*}$ for chitosan samples in $\mathrm{NaCl} /$ formic acid solution with different salt concentrations

\begin{tabular}{ccccccrr}
\hline Sample symbol & $\begin{array}{c}\text { The content of } \\
\text { amino groups } / \%\end{array}$ & $\bar{M}_{\mathrm{V}} \times 10^{-4}$ & \multicolumn{4}{c}{$C^{*} / \mathrm{w} / \mathrm{w} \%$} \\
\cline { 4 - 7 } & & $\mathrm{M}_{\text {salt }} / \mathrm{mol} \mathrm{L}^{-1}$ & 0 & 0.001 & 0.004 & 0.010 \\
\hline 1 & 7.06 & 58.0 & & 5 & 6 & 6 & 6 \\
3 & 8.06 & 34.9 & & 13 & 12 & 12 & 12 \\
\hline
\end{tabular}

lyte in formic acid. The critical concentration may be affected by electrostatic interaction among polymer chains, which seems responsible for low $C^{*}$ for this system at high molar mass. A preliminary experiment was done for the two higher molar mass samples (i. e., Nos. 1 and 3) to check electrostatic interactions by adding salt. The results are listed in Table III. $C^{*}$ did not increase with added salt concentration within experimental error, that is not expected by the theory of Stroobants $e t$ al. for $C^{*}$ of rodlike polyelectrolyte solutions. ${ }^{19}$ This may be attributed to the lower stiffness of chitosan, which is a semi-rigid polymer with $q=22 \mathrm{~nm}$.

\section{CONCLUSIONS}

Chitosan samples with molar mass from $1 \times 10^{4}$ to 58 $\times 10^{4}$ were prepared by depolymerization. In the higher molar mass region critical concentrations corresponded to predicted values of Flory theory. A significant raise of critical concentration with decrease of molar mass was observed, especially in the lower molar mass region. These results qualitatively agree with those calculated by the Khokhlov-Semenov theory based on typical semirigid chain. But in the low molar mass region they deviated from predicted values. This is explained by the inaccuracy of the second virial approximation basic to this theory at higher concentrations for the chains with small $q$. A logarithmic rule is suggested to describe the relationship between critical concentration forming liquid crystal phase and molar mass for chitosan/formic acid system.

Acknowledgment. The authors gratefully acknowl- edge the National Natural Science Foundation, China (No. 29974023) and Natural Science Foundation of Fujian, China (Z 9910006) for financial support.

\section{REFERENCES}

1. K. Ogura, T. Kanamoto, T. Sannan, K. Tanaka, and Y. Iwakura, "Chitin Chitosan Proc. Int. Conf. 2nd," Tottori, Japan, 1982, p 39.

2. K. Sakurai, T. Shibano, K. Kimura, and T. Takahashi, Sen-i Gakkaishi, 41, T-361 (1985).

3. M. Terbojevich, A. Cosani, G. Conio, E. Marsano, and E. Bianchi, Carbohydr. Res., 209, 251 (1991).

4. D. K. Rout, S. K. Pulapura, and R. A. Gross, Macromolecules, 26, 5999 (1993)

5. Y. Dong and Z. Li, Chin. J. Polym. Sci., 17, 65 (1999).

6. Y. Dong, Q. Yuan, Y. Wu, and M. Wang, J. Appl. Polym. Sci., 76, 2057 (2000).

7. J. Wang, Y. Dong, H. Liu, Q. Yuan, and X. Mei, Chem. J. Chin. Univ., 20, 474 (1999).

8. Z. Hu, R. Li, D. Wu, H. Liu, Y. Sun, and L. Wu, Acta Polymerica Sinica, 1, 46 (2000).

9. G. G. Allan and M. Peyron, Carbohydr, Res., 277, 257 (1995).

10. G. G. Allan and M. Peyron, Carbohydr, Res., 277, 273 (1995).

11. W. Wang, S. Buo, and W. Qiu, Science in China, Ser. B, 11, 1126 (1990).

12. T. Sannan, K. Kurita, K. Ogura, and Y. Iwakura, Polymer, 19, 458 (1978).

13. P. J. Flory, Proc. R. Soc. London, Ser. A, 234, 73 (1956).

14. A. R. Khokhlov and A. N. Semenov, Physica, 108A, 546 (1981).

15. A. R. Khokhlov and A. N. Semenov, Physica, 112A, 605 (1982).

16. L. Onsager and N. Y. Ann, Acad. Sci., 51, 627 (1949).

17. T. Odijk, Macromolecules, 19, 1220 (1986).

18. T. Itou and A. Teramoto, Macromolecules, 21, 2225 (1988).

19. A. Stroobants, H. N. W. Lekkerkerker, and T. Odijk, Macromolecules, 19, 2232 (1986). 